\title{
An UHF Thermal Coagulator for Achieving Hemostasis During Operations on Parenchymatous Organs
}

DOI: $10.17691 / \mathrm{stm} 2018.10 .2 .06$

Received January 15, 2017

G.G. Gorokhov, Head of the Unit';

L.V. Shkalova, MD, PhD, Head of the Department';

A.V. Strikovsky, PhD, Researcher';

S.V. Korobkov, Junior Researcher ${ }^{2}$;

M.E. Guschin, PhD, Head of the Laboratory2;

V.E. Zagainov, MD, DSc, Chief Specialist in Surgery'; Head of the Department of Surgical Diseases ${ }^{3}$

${ }^{1}$ Privolzhsky District Medical Center of Federal Medico-Biological Agency of Russia,

2 Nizhne-Volzhskaya naberezhnaya St., Nizhny Novgorod, 603001, Russia;

${ }^{2}$ Federal Research Center Institute of Applied Physics of the Russian Academy of Sciences, 46 Ulyanova St., Nizhny Novgorod, 603950, Russia;

${ }^{3}$ Privolzhsky Research Medical University, 10/1 Minin and Pozharsky Square, Nizhny Novgorod, 603005, Russia

The aim of the study is to assess the possibility of using an original UHF (ultra-high frequency) coagulator with an operating frequency of $2.45 \mathrm{GHz}$ to achieve hemostasis on the surfaces of parenchymatous organs.

Materials and Methods. The coagulator represents a coaxial line loaded on the antenna in the form of a monofilar Archimedean spiral. A maximal input power was $300 \mathrm{~W}$. Coagulator parameters were determined by the results of numerical simulation and laboratory tests at various loads. Coagulator work was studied on ovalbumin and liver parenchyma at various exposures. Biological tissue samples from the coagulation foci were histologically investigated.

Results. A coagulation focus with the diameter equal to the applicator diameter $(20 \mathrm{~mm})$ is formed during $10 \mathrm{~s}$ exposure at the maximum input power level. Tissue coagulation sufficiently uniform in depth is observed in the focus area.

Conclusion. The character of UHF field impact on the tissue does not depend on the degree of surface humidity. The impact area matches the diameter of the device working part allowing surgeons to plan the area of the appropriate exposure. The created complex is expected to find its application for hemostasis in parenchymatous hemorrhage.

Key words: hemostasis; parenchymatous organs; UHF applicator; UHF coagulator.

\section{Introduction}

Methods of achieving hemostasis can be conditionally divided into mechanical, chemical, and physical. The mechanical techniques are employed only if vessels of medium or large caliber are damaged and in this case clips, ligatures, suture material, etc. are used. Chemical methods involve using vasoconstrictor agents increasing blood clotting [1]. Gelaspon, for example, representing a sponge from a foamed gelatin accelerates hemostasis, as contact with gelatin releases thrombocytes. In capillary and parenchymatous bleedings, carbazochrome is also used, which decreases vessel permeability and normalizes blood microcirculation. In general, chemical agents are rarely used for local blood control due to their insufficient efficacy.

Physical or thermal methods of hemostasis based on low or high-temperature technologies have found wide application. However, neither of these physical methods is devoid of shortcomings. Cryogenic methods realized at the temperatures from -20 to $-180^{\circ} \mathrm{C}$ require application of a significant number of probes different in size and configuration, though it is impossible to control the size and form of the tissue coagulation zone [2]. Monopolar or bipolar electrocoagulators employing hightemperature technologies cause formation of coagulation eschar, which is characterized by a great risk of rejection with the following bleeding recurrence. Besides, electric current running through the patient's body can cause electrolysis of blood and biological tissues, which may have an unfavorable effect on the recovery dynamics. Therefore, in the process of hemostasis, a full discharge current has to be limited at the expense of the volume and area of the treated surface. Moreover, when working, coagulators often cause burn injuries in the area of the base electrode contact requiring additional treatment [3].

As of today, plasma coagulation seems to be the most promising method of bleeding control. The technique makes it possible to provide "a clean operative field"

Corresponding author: Gleb G. Gorokhov, e-mail: gorochov@gmail.com 
removing free tissue fragments and blood clots by a directional flow of argon plasma. A drawback of plasma coagulators is uncontrolled necrosis of the treated tissue and the risk of burn with the perforation of a hollow organ. Another drawback is formation of coagulation eschar, which prevents further hemostasis [4].

A sufficiently effective method of bleeding arrest, including bleeding from the parenchymatous organs, is laser photocoagulation possessing a number of advantages over electrocoagulation: no mechanical contact and, consequently, no sticking of the coagulated tissues to the electrode and their burning; strict dosing and uniform distribution of energy within a light spot; continuous visual control since the bleeding area is never covered with the electrode; absence of electrical current affecting the tissues, and localization of necrobiotic alterations in the zone of thermal impact.

A major disadvantage of this method is a small laser beam area acting on the treated tissue (about 1-2 $\mathrm{mm}^{2}$ ) making bleeding control over a vast surface very difficult $[5,6]$.

Fulguration, also called spray-coagulation, is one more physical technique. A large-amplitude, highfrequency voltage forms spark discharges having no direct contacts with a biological tissue. The discharges cause extreme temperature rise on the tissue surface and destroy it resulting in superficial coagulation, eschar formation, and tissue carbonization. Spray-coagulation is used for hemostasis of large surfaces, for example, in severe hemorrhages (so-called oozing surfaces). In this case, a formed burn eschar aggravates healing leading to bleeding recurrence [7].

Thus, for the present, there is not a single reliable method of bleeding control on a biological tissue surface and, therefore, the search of novel technical solutions is of vital importance.

This work is devoted to the study of bulk and surface phenomena occurring under the local exposure of parenchymatous tissues to high-intensity UHF field to arrest bleedings from parenchymatous organs such as liver, kidneys, and spleen during surgical interventions. Tissue coagulation of these organs features constant formation of blood film on the surface, and all known physical methods of arresting bleeding from the parenchymatous organs require their preliminary draining.

The aim of the study is to assess the possibility of using an original UHF coagulator with an operating frequency of $2.45 \mathrm{GHz}$ to achieve hemostasis on the surfaces of parenchymatous organs.

\section{Materials and Methods}

On the basis of the prototype of the medical UHF complex for a thermal effect on biological tissues [8-11] developed in 2011, a model of UHF coagulator was created for the work with parenchymatous organs (Figure 1). This device provides maximal output power

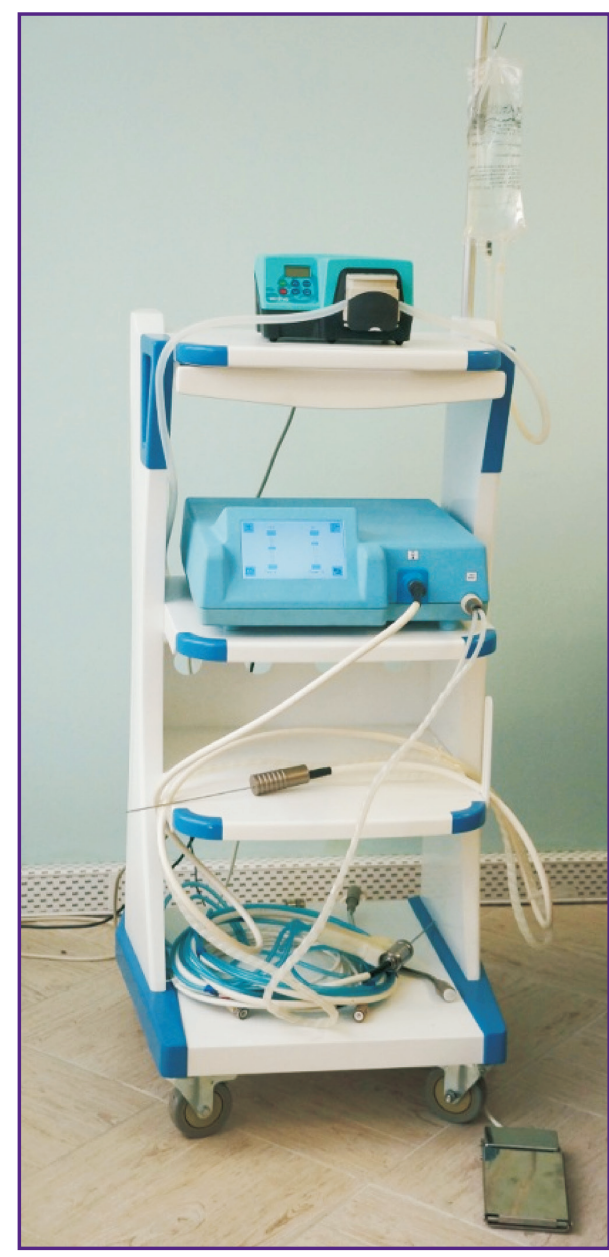

Figure 1. A medical UHF complex for thermal coagulation of parenchymatous tissues

of $300 \mathrm{~W}$ at an operating frequency of $2.45 \mathrm{GHz}$, has an improved ergonomics with an intuitive control menu, programmed modes of action on biological tissues by the form of UHF field envelope and an average power level.

Exposure of the biological tissues to the electromagnetic field results in volume-distributed conversion of electromagnetic energy to the thermal one providing an effective bulk heating. The structure of the temperature field depends primarily on the structure of thermogenesis determined by the operating frequency, antenna construction, and biophysical characteristics of the tissue itself. Transition to a higher frequency (to UHF range) relative to $\mathrm{HF}$ range of traditionally used coagulators allows the increase of the power absorbed by the substance. At the same input power, heating of the biological tissue in the UHF range occurs more effectively, hence, the speed of heating the treated area is greater. As the result, there is a possibility of a quick local effect on the vessels, which is accompanied by coagulation of albumin and its "sealing". In particular, 
when a bleeding surface is exposed to a thermal impact of the UHF field, a 2-3 mm (or more) coagulation layer is formed depending on the organ and its structure. A reliable hemostasis of the damaged organ, including the period of operative intervention, is achieved independently of the presence of biological fluid on its surface.

Figure 2 shows the applicator for supplying UHF power to a biological tissue. Selection of the configuration and antenna parameters (Figure 3 ) was preceded by experimental optimality investigations and physical analysis of the electrodynamic near-field system in the mode of UHF power action on the biological tissue. In terms of design, the antenna used for coagulation is made in the form of a plane monofilar Archimedean spiral providing a wide-aperture effect of UHF field on the biological tissue. Coaxial radiofrequency cable carries UHF power to the antenna. The greatest intensity of UHF field is achieved opposite the spiral wire. The electromagnetic field of smaller intensity between the spiral turns facilitates the equalization of the biological tissue temperature across the impact spot. To suppress the reflected UHF wave propagating along the cable armature towards the generator as well as to attenuate the parasitic UHF oscillations at the air-biological tissue interface, two coaxial quarter-wave resonators are used. A Teflon cover on the working part of the applicator protects the antenna and resonators against fluid penetration and prevents sticking of the biological tissue to the applicator. The applicator diameter is $20 \mathrm{~mm}$.

The applicator parameters were chosen and justified by the results of numeric simulation of the electrodynamic system comprising radiofrequency cable, antenna system, and biological tissue with realistic parameters of conductivity and dielectric permittivity. According to the calculations presented in Figure 4, the maximal amplitude of the applicator near UHF field is reached at a distance of several millimeters from the spiral before the Teflon cover. A cross-section of the electric UHF field intensity modulus at a distance of $2 \mathrm{~mm}$ from the antenna is shown in Figure 5. Field distribution follows the form of the spiral and, accordingly, the heat input occurs in this area, however, due to the thermal conductivity of the biological tissue the temperature equalizes rather quickly over the applicator aperture. tissues:
Measurements of the UHF power coefficient of reflection from the applicator in a free space (without a load) and applied to the biological tissue (with a load) are presented in Figure 6. At the operating frequency $(2.45 \mathrm{GHz})$, the difference in the reflection coefficients between the loaded and unloaded applicator is $16 \mathrm{~dB}$, which indicates to the optimal mode of matching the coagulator with the biological tissue.

The possibilities of using the applicator for coagulation of parenchymatous tissues were investigated on ovalbumin and the right liver lobe removed from the patient with colorectal metastases. The liver tissues were exposed to two power modes of UHF generators: $75 \%(225 \mathrm{~W})$ and $100 \%(300 \mathrm{~W})$ with 2, 5, 10, and $15 \mathrm{~s}$ exposure in each mode. The material taken from the liver coagulation foci was marked and sent for histological investigations.

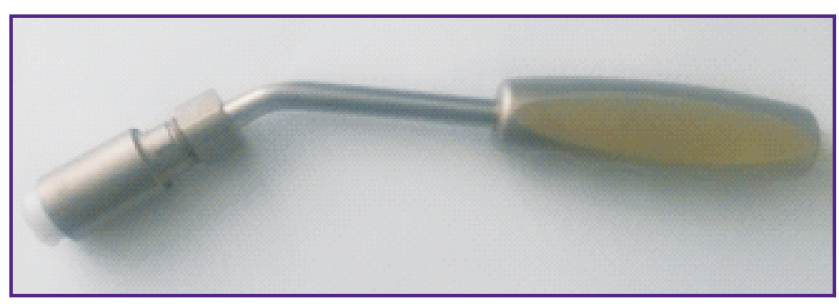

Figure 2. General appearance of the applicator for UHF thermal coagulation

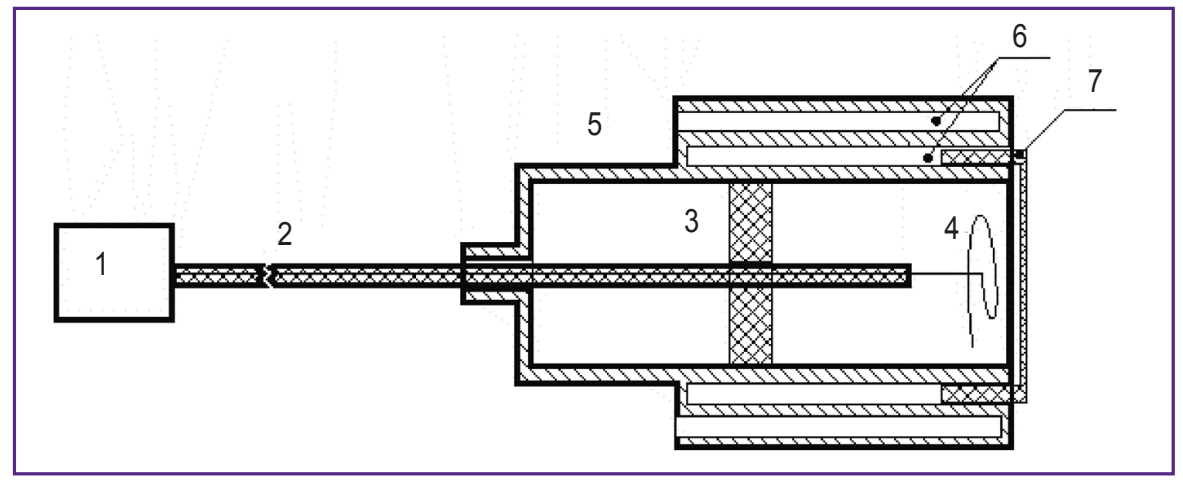

Figure 3. Near-field antenna system for UHF thermal coagulation of biological

(1) UHF generator; (2) radiofrequency cable; (3) centering cable support; (4) monofilar Archimedean spiral; (5) coagulator body; (6) quarter-wave resonators; (7) dielectric cover

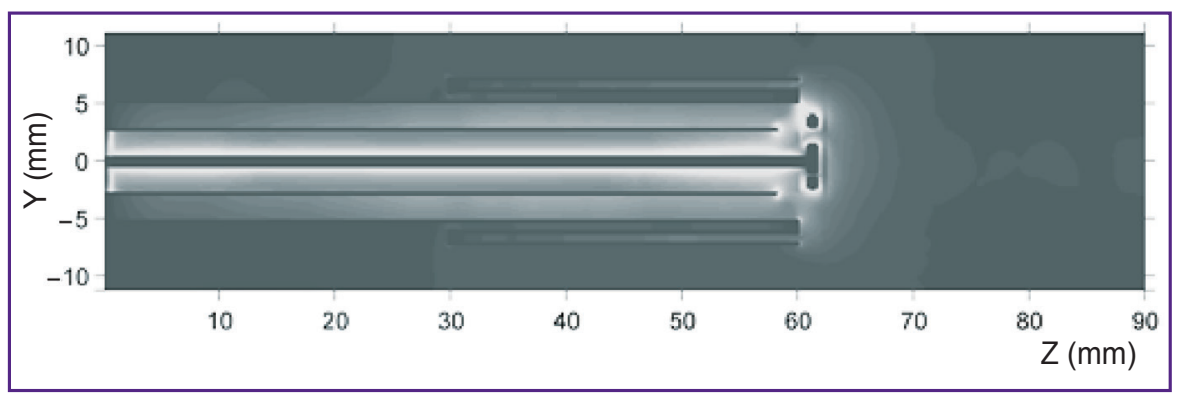

Figure 4. Calculated distribution of the electric UHF field intensity modulus in the longitudinal section of the coaxial line loaded on the applicator 


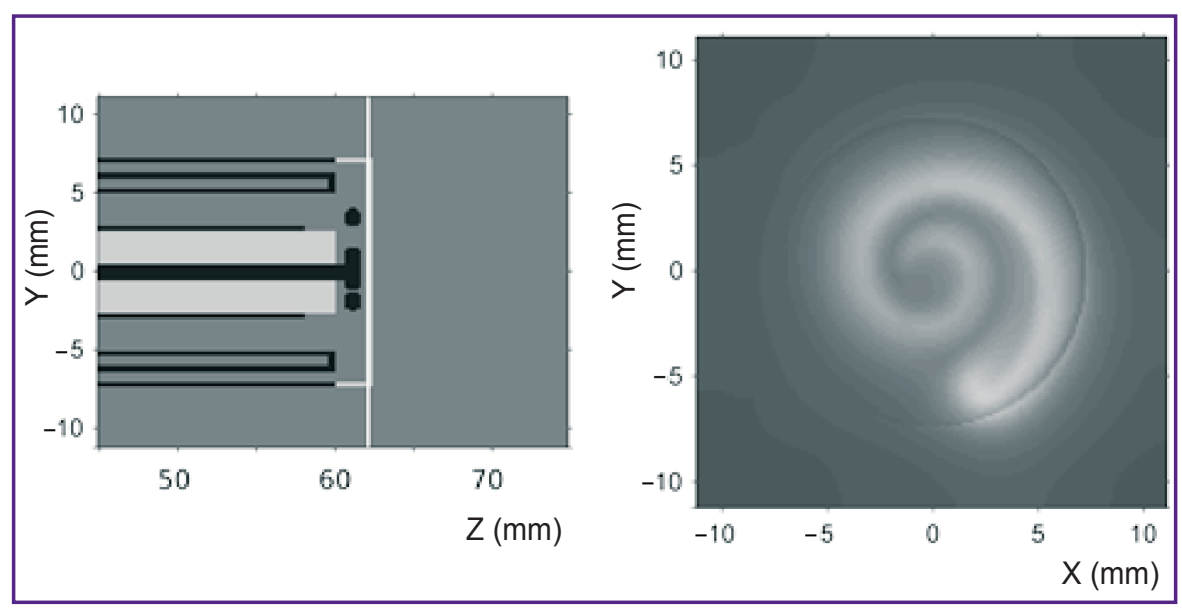

Figure 5. Transverse structure of the electric UHF field modulus in the working zone of the applicator (calculation)

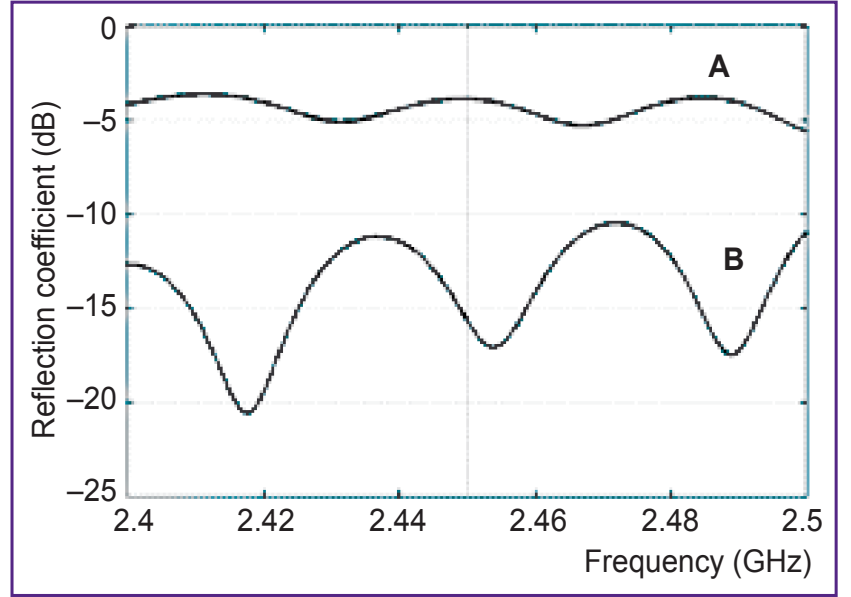

Figure 6. UHF power coefficient of reflection from unloaded (A) and loaded (B) applicator (measurement)

\section{Results and Discussion}

Figure 7 illustrates the results of the applicator action on the ovalbumin. The coagulated albumin is uniform in thickness and aperture, which has the form of a circle with the diameter equal to that of the applicator.

A photograph of the liver with coagulation foci obtained at various exposures is presented in Figure 8. Histological samples were taken (Figure 9). The data of histological investigations enabled us to obtain the dimensional parameters of the coagulation foci on the liver depending on the exposure time and power (Figure 10).

Referring to Figure 8, it is seen that the form of the coagulation foci approaches the calculated distributions of the electromagnetic fields (see Figures 4 and 5). Visually, the greatest alterations are observed in the center of the impact spot. The comparison of the histological samples demonstrates a distinct dependence of the alteration area dimensions on the impact time and power (see Figure 10). There are no data on the changes in the transverse focus structure

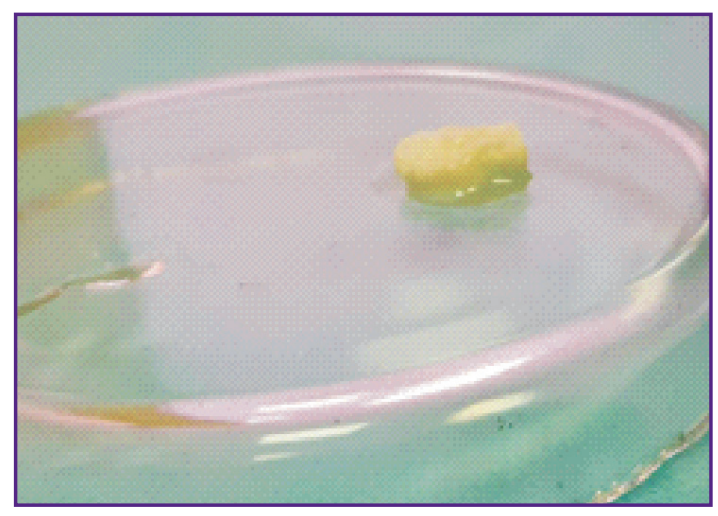

Figure 7. Exposure of the ovalbumin to the UHF coagulator

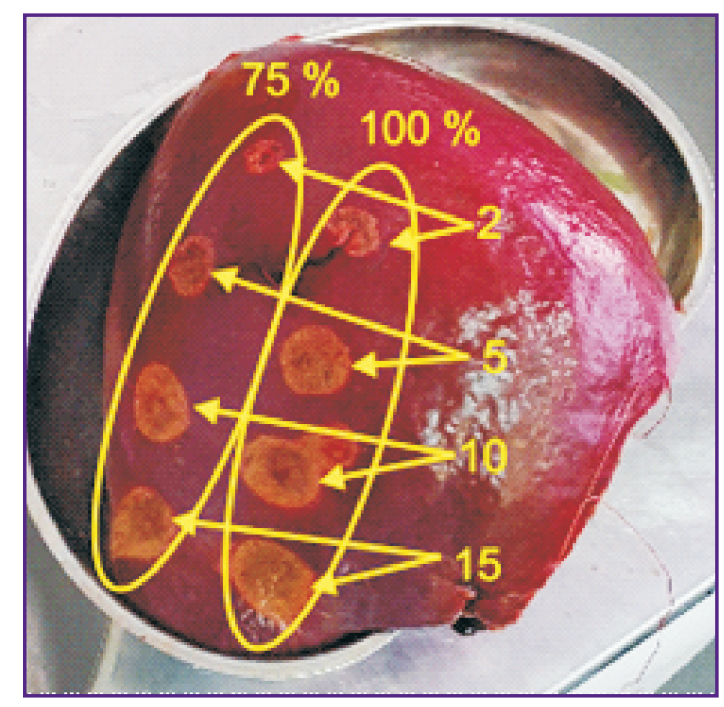

Figure 8. Right liver lobe subjected to UHF coagulator treatment:

2, 5, 10, $15 \mathrm{~s}$ exposure, $75 \%$ and $100 \%$ UHF generator power

in the first $2 \mathrm{~s}$ but beginning with the third second the focus diameter is monotonically increasing and reaches in $10 \mathrm{~s}$ the value close to the applicator diameter. The 
Figure 9. Histological liver samples (1-3) treated with the UHF coagulator in the mode of $100 \%$ generator power; $15 \mathrm{~s}$ impact time
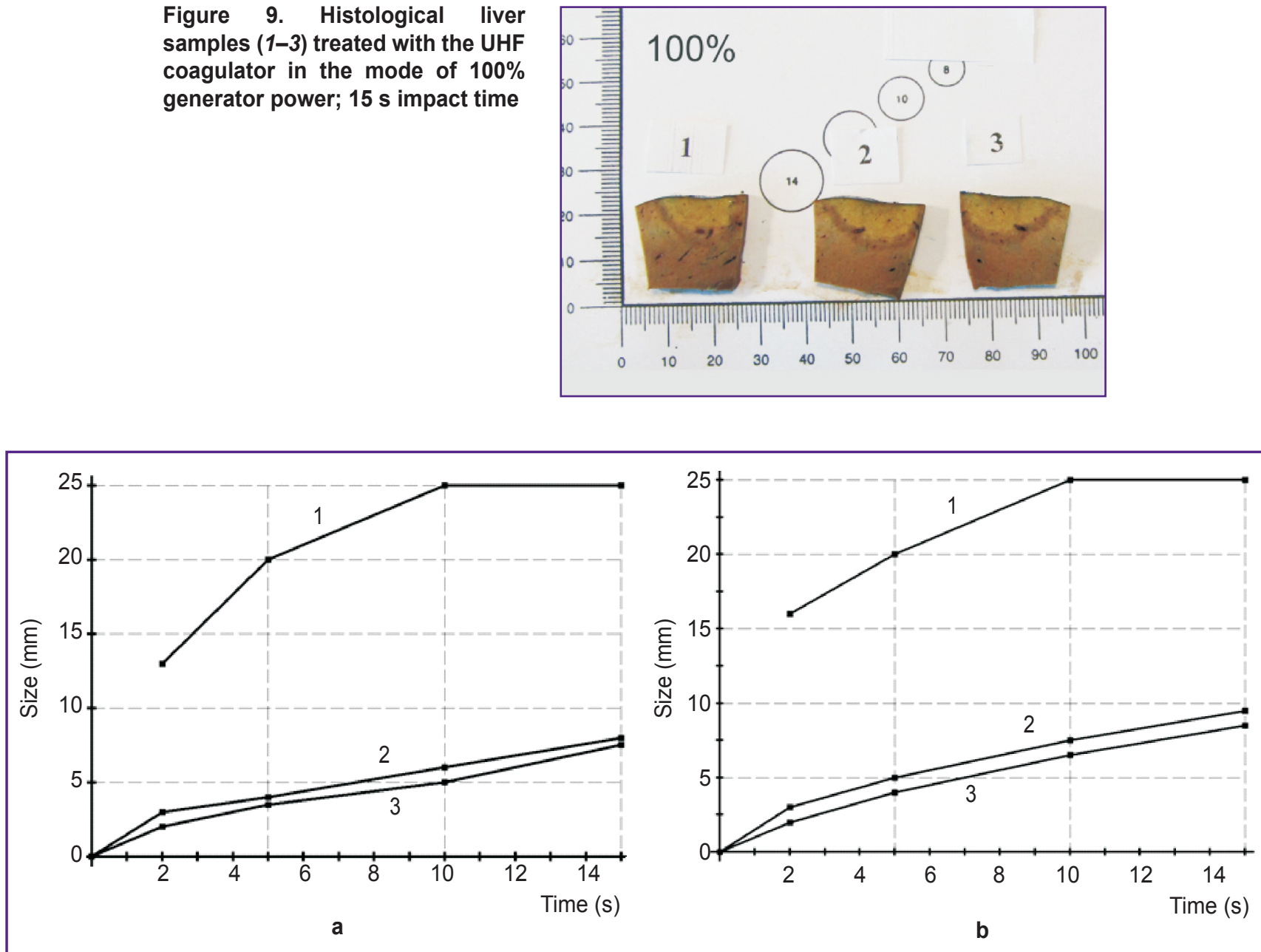

Figure 10. Results of the UHF coagulator impact on the liver depending on the exposure time:

(a) in $75 \%$ power mode; (b) in 100\% power mode; (1) focus diameter; (2) focus depth in the center; (3) focus depth at the periphery

diameter of the focus does not change till the fifteenth second of the impact. Such dynamics is defined by the configuration of the antenna electromagnetic field and alterations in the absorbing properties of the biological tissue in the process of exposure. During the first seconds of tissue heating, a focus of visible coagulation changes having delineated boundaries is formed. Though the UHF field is not uniform over the applicator aperture, the field of heating is homogeneous enough within the spot due to high thermal tissue conductivity. Starting approximately with the tenth second, the visible size of the impact area stops increasing and becomes equal to the diameter of the device working part, and is determined by the distribution of the electric field. As to the coagulation depth, its value in the center of the impact spot and at the periphery differs for each fixed moment of time, though the difference does not exceed $20 \%$. Beginning with second 2, the border of macroscopic changes goes deeper into the biological tissue at almost a constant speed from $0.35 \mathrm{~mm} / \mathrm{s}$ in the mode of $75 \%$ power to $0.45 \mathrm{~mm} / \mathrm{s}$ in the $100 \%$ power mode. At the same time, the coagulation depth for the two power modes differs by no more than $10-15 \%$ at maximum exposure time (15 s).

\section{Conclusion}

The findings obtained in the course of the experiments on coagulation of the ovalbumin and liver parenchyma using UHF energy show that the structure of the temperature field depends primarily on the structure of thermogenesis in the tissues, which is determined by the field frequency, radiating element construction, and biophysical characteristics of the tissue itself. The impact character does not depend on the degree of surface humidity, herewith the impact zone matches the diameter of the working part of the device. A sharp border of the zone of coagulation alterations enables surgeons to plan the impact area. The results obtained in our experiment prove the possibility of using the described system for hemostasis in parenchymatous bleeding. 
Study funding. The work was supported by the Russian Foundation of Basic Research (grant No.15-4202348-r_povolzhye_a).

Conflicts of interest. The authors declare absence of financial and any other conflicting interests capable of exerting influence on their work.

\section{References}

1. Safronova E.U., Nushko K.M., Alekseev B.Y., Kalpinskiy A.S., Polyakov V.A., Kaprin A.D. Methods of performing of hemost asis during kidney resection. Research'n Practical Medicine Journal 2016; 3(1): 58-65, https://doi. org/10.17709/2409-2231-2016-3-1-8.

2. Aleksandrov V.V., Maskin S.S., Igolkina L.A., Ermolaeva N.K. Prospects for the use of local cryohemostasis in cases of liver and splenic trauma. Kubanskiy nauchnyy meditsinskiy vestnik 2013; 7(142): 45-51.

3. Pakhlevanyan V.G., Kolesnikov S.A. Electrical coagulation hemostasis, advantages and disadvantages. Nauchnye vedomosti Belgorodskogo gosudarstvennogo universiteta. Seriya: Meditsina. Farmatsiya 2016; 33(5): 5-9.

4. Nomura Y., Takamatsu T., Kawano H., Miyahara H., Okino A., Yoshida M., Azuma T. Investigation of blood coagulation effect of nonthermal multigas plasma jet in vitro and in vivo. J Surg Res 2017; 219: 302-309, https://doi. org/10.1016/j.jss.2017.06.055.

5. Kirschbaum A., Rexin P., Pehl A., Bartsch D., Quint K. Laser resection of lung tissue: heat accumulation from adjacent laser application and how to cool it down.
Thorac Cardiovasc Surg 2014; 62(4): 363-368, https://doi. org/10.1055/s-0033-1358780

6. Shapkin Yu.G., Maslyakova G.N., Kapralov S.V., Uryadov S.E., Ivanov R.Yu., Grishaev V.A. Laser photocoagulation of the bleeding gastric and duodenal ulcer. Khirurgiya. Zhurnal im. N.I. Pirogova 2006; 5: 23-25.

7. Hilal Z., Mavrommati G., Foerster C., Rezniczek G.A., Hefler L.A., Tempfer C.B. Spray versus forced coagulation in large loop excision of the transformation zone: a randomized trial. J Low Genit Tract Dis 2016; 20(2): 169-173, https://doi. org/10.1097/lgt.0000000000000177.

8. Strikovskiy A.V., Kostrov A.V., Zagainov V.E., Sudakov M.A., Odzerikho D.A. Device for thermocoagulation of biological tissues by super high frequencies. Patent RU 2481080. 2011.

9. Zagainov V.E., Kostrov A.V., Strikovsky A.V., Gorokhov G.G., Vasenin S.A., Shkalova L.V., Rykhtick P.I., Shatokhina I.V., Bugrova M.L., Avdonin S.N. The use of a local effect of a superhigh frequency (SHF) energy for the liver tumor thermal destruction (in clinic). Sovremennye tehnologii $v$ medicine 2011; 1: 29-33.

10. Zagainov V.E., Kostrov A.V., Strikovskij A.V., Gorokhov G.G., Yanin D.V., Vasenin S.A., Shkalova L.V., Bugrova M.L., Snopova L.B., Atduev V.A. Experimental substantiation of the new method of thermal destruction of tumors of parenchymatous organs by the local action of the energy of superhigh frequency. Medicinskij al'manah 2011; 18(5): 60-66.

11. Zagainov V.E., Gorokhov G.G., Zarechnova N.V., Rykhtik P.I., Vasenin S.A., Sudakov M.A., Kostrov A.V., Strikovskii A.V. Surgical treatment of colorectal cancer liver metastases with microwave thermoablation. Khirurgiya. Zhurnal im. N.I. Pirogova 2011; 8: 61-66. 